\title{
AUTHORS CONTRIBUTING TO THE JEPSON MANUAL, SECOND EDITION
}

James D. Ackerman, University of Puerto Rico, Río Piedras

Robert P. Adams, Baylor University, Waco, Texas

Carlos Aedo, Real Jardín Botánico, Madrid, Spain

Susan G. Aiken, Canadian Museum of Nature, Ottawa, Ontario

Marisa Alarcón, Real Jardín Botánico, Madrid, Spain

Lawrence A. Alice, Western Kentucky University, Bowling Green

Charles Allen, Colorado State University, Fort Collins

Geraldine A. Allen, University of Victoria, British Columbia, Canada

Robert Lee Allen, Rancho Santa Ana Botanic Garden, Claremont, California

Kelly W. Allred, New Mexico State University, Las Cruces

Ihsan A. Al-Shehbaz, Missouri Botanical Garden, St. Louis

Edward F. Anderson†ं, Whitman College, Walla Walla, Washington

Katarina Andreasen, Uppsala University, Sweden

George W. Argus, Canadian Museum of Nature, Ottawa, Ontario, Canada

Wayne P. Armstrong, Palomar College, San Marcos, California

T.A. Atkinson, Burlington, North Carolina

Daniel F. Austin, Arizona-Sonora Desert Museum, Tucson

Deborah Engle Averett, Austin, Texas

Tina Ayers, Northern Arizona University, Flagstaff

Susan J. Bainbridge, University of California, Berkeley

Gary I. Baird, Brigham Young University-Idaho, Rexburg

$\dagger$ Deceased
John R. Baird, Ohio Department of Transportation, Columbus

Marc Baker, Arizona State University, Tempe

Riccardo M. Baldini, Università degli Studi, Firenze, Italy

Bruce G. Baldwin, University of California, Berkeley

Peter W. Ball, University of Toronto, Mississauga, Ontario, Canada

Theodore M. Barkley†, Botanical Research Institute of Texas, Fort Worth

Kerry Barringer, Brooklyn Botanic Garden, New York

Jim A. Bartel, United States Fish and Wildlife Service, Carlsbad, California

Robyn Battaglia, Pleasanton, California

Bernard R. Baum, Agriculture and Agri-Food Canada, Ottawa, Ontario, Canada

Randall J. Bayer, University of Memphis, Tennessee

Charles D. Bell, University of New Orleans, Louisiana

Hester L. Bell, Rancho Santa Ana Botanic Garden, Claremont, California

Robin Bencie, Humboldt State University, Arcata, California

Nuri Benet-Pierce, San Diego State University, California

Mark W. Bierner, The University of Texas at Austin; The University of Arizona, Tucson

John Bleck, University of California, Santa Barbara

David Bogler, Missouri Botanical Garden, St. Louis

Allan J. Bornstein, Southeast Missouri State University, Cape Girardeau

Steve Boyd, Rancho Santa Ana Botanic Garden, Claremont, California

David M. Brandenburg, The Dawes Arboretum, Newark, Ohio

Joshua M. Brokaw, Washington State University, Pullman 
Luc Brouillet, Université de Montréal, Québec, Canada

Gregory K. Brown, University of Wyoming, Laramie

R.K. Brummitt, Royal Botanic Gardens, Kew, Richmond, United Kingdom

Mark S. Brunell, University of the Pacific, Stockton, California

Christopher S. Campbell, University of Maine, Orono

Gerald D. Carr, Oregon State University, Corvallis

Robert L. Carr, Eastern Washington University, Cheney

Rosa Cerros-Tlatilpa, Universidad Autónoma del Estado de Morelos, Cuernavaca, Mexico

Adolf Ceska, Victoria, British Columbia, Canada

Oldriska Ceska, Victoria, British Columbia, Canada

Henrietta L. Chambers, Oregon State University, Corvallis

Kenton L. Chambers, Oregon State University, Corvallis

Raymund Chan, University of California, Berkeley

Anita F. Cholewa, University of Minnesota, St. Paul

T.I. Chuang $\dagger$, Illinois State University, Normal

Timothy Chumley, The University of Texas at Austin

Curtis Clark, California State Polytechnic University, Pomona

Lynn G. Clark, Iowa State University, Ames

W.D. Clark, Arizona State University, Tempe

Steven E. Clemants $\dagger$, Brooklyn Botanic Garden, New York

Ronald A. Coleman, The University of Arizona, Tucson

J. Travis Columbus, Rancho Santa Ana Botanic Garden and Claremont Graduate University, California

Alison E.L. Colwell, National Park Service, Yosemite National Park, El Portal, California

Steven A. Conley, Redding, California

H.E. Connor, University of Canterbury, Christchurch, New Zealand

Lincoln Constance $\uparrow$, University of California, Berkeley

Mihai Costea, Wilfrid Laurier University, Waterloo, Ontario, Canada

Lyn A. Craven, Australian National Herbarium, Canberra, Australia

William J. Crins, Ontario Ministry of Natural Resources, Peterborough, Ontario, Canada

Michael Curto, California Polytechnic State University, San Luis Obispo
Thomas F. Daniel, California Academy of Sciences, San Francisco

S.J. Darbyshire, Agriculture and Agri-Food Canada, Ottawa, Ontario, Canada

Jerrold I. Davis, Cornell University, Ithaca, New York

W.S. Davis, Louisville, Kentucky

Alva G. Day, California Academy of Sciences, San Francisco

Sarah De Groot, Rancho Santa Ana Botanic Garden and Claremont Graduate University, California

Alfonso Delgado-Salinas, Universidad Nacional Autónoma de México, México City

Lauramay T. Dempstert, University of California at Berkeley

Melinda F. Denton†, University of Washington, Seattle

James C. Dice, Natural History Museum, San Diego, California

John E. Ebinger, Eastern Illinois University, Charleston

Patrick E. Elvander†, University of California at Santa Cruz

Mark A. Elvin, United States Fish and Wildlife Service, Ventura, California

Barbara Ertter, University of California, Berkeley

Frederick Essig, University of South Florida, Tampa

Dwayne Estes, Austin Peay State University, Clarksville, Tennessee

Donald R. Farrar, Iowa State University, Ames

Carolyn J. Ferguson, Kansas State University, Manhattan

Wayne R. Ferren, Jr., Rutgers, The State University of New Jersey, New Brunswick

Peggy L. Fiedler, University of California Natural Reserve System, Oakland

Petra Foerster, Fredericton, New Brunswick, Canada

Bruce A. Ford, University of Manitoba, Winnipeg, Canada

Robert W. Freckmann, University of WisconsinStevens Point

Peter W. Fritsch, California Academy of Sciences, San Francisco

Paul A. Fryxell $\dagger$, The University of Texas at Austin

John C. Game, University of California, Berkeley

Fred R. Ganders, University of British Columbia, Vancouver, Canada

Laura M. Garrison, Brown University, Providence, Rhode Island 
John F. Gaskin, United States Department of Agriculture, Agricultural Research Service, Sidney, Montana

Peter Goldblatt, Missouri Botanical Garden, St. Louis

Douglas H. Goldman, Harvard University, Cambridge, Massachusetts

L.D. Gottlieb, University of California, Davis

David Gowen, University of California, Berkeley

Shirley A. Graham, Missouri Botanical Garden, St. Louis

Craig W. Greenet, College of the Atlantic, Bar Harbor, Maine

Brenda J. Grewell, United States Department of Agriculture, Agricultural Research Service, Davis, California; University of California, Davis

James R. Griffin†, University of California at Berkeley

James W. Grimes, New York Botanical Garden, Bronx

Evanielis U. Grissom, University of Illinois, UrbanaChampaign

C. Matt Guilliams, University of California, Berkeley

Erich Haber, Canadian Museum of Nature, Ottawa, Ontario, Canada

J. Robert Haller, Santa Barbara Botanic Garden; University of California, Santa Barbara

Richard R. Halse, Oregon State University, Corvallis

Gary L. Hannan, Eastern Michigan University, Ypsilanti

Debra R. Hansen, The University of Texas at Austin

Danica T. Harbaugh, University of California, Berkeley

Raymond M. Harley, Royal Botanic Gardens, Kew, Richmond, United Kingdom

Ronald L. Hartman, University of Wyoming, Laramie

Michael J. Harvey, Dalhousie University, Halifax, Canada; Victoria, British Columbia, Canada

Kristen E. Hasenstab-Lehman, Rancho Santa Ana Botanic Garden and Claremont Graduate University, California

Richard L. Hauke, University of Rhode Island, Kingston and Atlanta, Georgia

Frank G. Hawksworth $\dagger$, United States Forest Service, For Collins, Colorado

Robert R. Haynes, The University of Alabama, Tuscaloosa

Lawrence R. Heckard $\dagger$, University of California, Berkeley
Alice Long Heikens, Franklin College, Indiana

C. Barre Hellquist, Massachusetts College of Liberal Arts, North Adams

James Henrickson, The University of Texas at Austin

William J. Hess, The Morton Arboretum, Lisle, Illinois

Steven R. Hill, Illinois Natural History Survey, University of Illinois, Urbana-Champaign

Matthew H. Hils, Hiram College, Ohio

Andrew L. Hipp, The Morton Arboretum, Lisle, Illinois; The Field Museum, Chicago, Illinois; University of Wisconsin-Madison

Peter C. Hoch, Missouri Botanical Garden, St. Louis

Carol A. Hoffman, University of Georgia, Athens

Scott A. Hodges, University of California, Santa Barbara

Noel H. Holmgren, New York Botanical Garden, Bronx

Charles N. Horn, Newberry College, South Carolina

G. Frederic Hrusa, California Department of Food and Agriculture, Sacramento

Larry Hufford, Washington State University, Pullman

Stefanie M. Ickert-Bond, University of Alaska, Fairbanks

Duane Isely†ं, Iowa State University, Ames

Judith A. Jernstedt, University of California, Davis

Dale E. Johnson, Seattle, Washington

George P. Johnson, Arkansas Tech University, Russellville

Leigh A. Johnson, Brigham Young University, Provo, Utah

James D. Jokerst $\dagger$, Jones and Stokes Associates, Sacramento, California

William Jones, California State University, Los Angeles

Walter S. Judd, University of Florida, Gainesville

Steve Junak, Santa Barbara Botanic Garden, California

Glenn Keator, Sebastopol, California

Jon E. Keeley, Rancho Santa Ana Botanic Garden, Claremont, California

David J. Keil, California Polytechnic State University, San Luis Obispo

Ronald B. Kelley, Eastern Oregon University, La Grande

Sylvia Kelso, Colorado College, Colorado Springs

Kim R. Kersh, University of California, Berkeley

Carrie Kiel, Rancho Santa Ana Botanic Garden and Claremont Graduate University, California 
Ruth E.B. Kirkpatrick, University of California, Berkeley

Jan Kirschner, Institute of Botany, Academy of Sciences, Průhonice, Czech

Genevieve J. Kline, Northern Illinois University, DeKalb

Jason A. Koontz, Augustana College, Rock Island, Illinois

Daryl Koutnik, Impact Sciences, Inc., Camarillo, California

Kathleen A. Kron, Wake Forest University, WinstonSalem, North Carolina

Job Kuijt, University of Victoria, British Columbia, Canada

John C. La Duke, University of North Dakota, Grand Forks

Leslie R. Landrum, Arizona State University, Tempe

Meredith A. Lane, Reston, Virginia

Richard V. Lansdown, Ardeola Environmental Services, Stroud, United Kingdom

Robert Lauri, Rancho Santa Ana Botanic Garden, Claremont, California

Matt Lavin, Montana State University, Bozeman

Thomas Lemieux, University of Colorado, Boulder

Gordon Leppig, California Department of Fish and Game, Eureka

Donald H. Les, University of Connecticut, Storrs

Deborah Q. Lewis, Iowa State University, Ames

Harlan Lewist, University of California, Los Angeles

Richard A. Lis, University of California at Berkeley

R. John Little, Sycamore Environmental Consultants, Inc., Sacramento, California

Lúcia G. Lohmann, Universidade de São Paulo, Brazil

Timothy K. Lowrey, University of New Mexico, Albuquerque

Karen Lu, Humboldt State University, Arcata, California

B.H. Macmillan, Landcare Research, Lincoln, New Zealand

L.K. Magrath $\uparrow$, University of Science and Arts of Oklahoma, Chickasha

Alison M. Mahoney, Minnesota State University, Mankato

Staci Markos, University of California, Berkeley

Joy Mastrogiuseppe, Washington State University, Pullman

Mark H. Mayfield, Kansas State University, Manhattan
Stephen Ward McCabe, University of California, Santa Cruz

Elizabeth McClintock $\dagger$, University of California, Berkeley

Joshua R. McDill, The University of Texas at Austin

Robert J. McKenzie, Rhodes University, Grahamstown, South Africa

Michelle M. McMahon, The University of Arizona, Tucson

Dale W. McNeal, University of the Pacific, Stockton, California

T. Lawrence Mellichamp, University of North Carolina, Charlotte

Michael R. Mesler, Humboldt State University, Arcata, California

Timothy C. Messick, Jones and Stokes Associates, Sacramento, California

Constance I. Millar, United States Department of Agriculture, Forest Service, Pacific Southwest Research Station, Albany, California

John M. Miller, University of California, Berkeley Abigail J. Moore, University of California, Berkeley Michael O. Moore, University of Georgia, Athens

John S. Mooring, Santa Clara University, California

James D. Morefield, Nevada Natural Heritage Program, Carson City

David R. Morgan, University of West Georgia, Carrollton

Nancy R. Morin, Flora of North America, Point Arena, California

Andy Murdock, University of California, Berkeley

Carmen Navarro, Universidad Complutense, Madrid, Spain

Michael H. Nee, New York Botanical Garden, Bronx

Elizabeth Chase Neeset, University of California, Berkeley

John B. Nelson, University of South Carolina, Columbia

Guy L. Nesom, Fort Worth, Texas

Bryan D. Ness, Pacific Union College, Angwin, California

Lorin I. Nevling, Jr., Illinois Natural History Survey, University of Illinois, Urbana-Champaign

Gilberto Ocampo, California Academy of Sciences, San Francisco

Sang-Hun Oh, Cornell University, Ithaca, New York

Richard G. Olmstead, University of Washington, Seattle

Robert Ornduff $\dagger$, University of California, Berkeley 
Elizabeth L. Painter, Santa Barbara Botanic Garden, California

Bruce D. Parfitt†, The University of Michigan-Flint

Michael S. Park, University of California, Berkeley

V. Thomas Parker, San Francisco State University, California

Robert Patterson, San Francisco State University, California

Paul M. Peterson, Smithsonian Institution, Washington, District of Columbia

James B. Phipps, The University of Western Ontario, London, Canada

Michael B. Piep, Utah State University, Logan

J. Chris Pires, University of Missouri, Columbia

Duncan M. Porter, Virginia Polytechnic Institute and State University, Blacksburg

J. Mark Porter, Rancho Santa Ana Botanic Garden and Claremont Graduate University, California

Daniel Potter, University of California, Davis

A. Michael Powell, Sul Ross State University, Alpine, Texas

Robert E. Preston, ICF International, Sacramento, California

Barry Prigge, University of California, Los Angeles

James S. Pringle, Royal Botanical Gardens, Hamilton, Ontario, Canada

Charles F. Quibell, Sonoma State University, Rohnert Park, California

Richard K. Rabeler, The University of MichiganAnn Arbor

Jon Rebman, San Diego Natural History Museum, California

John R. Reedert, The University of Arizona, Tucson

Nancy F. Refulio-Rodriguez, Rancho Santa Ana Botanic Garden, Claremont, California

James L. Reveal, Cornell University, Ithaca, New York

Barry A. Rice, University of California, Davis

Matt Ritter, California Polytechnic State University, San Luis Obispo

Joseph R. Rohrer, University of Wisconsin-Eau Claire

Thomas J. Rosatti, University of California, Berkeley

Jeffery M. Saarela, Canadian Museum of Nature, Ottawa, Ontario, Canada

Andrew C. Sanders, University of California, Riverside

John O. Sawyer, Jr., Humboldt State University, Arcata, California

H. Jochen Schenk, California State University Fullerton
John J. Schenk, Washington State University, Pullman

Robert L. Schlising, California State University, Chico

Lisa M. Schultheis, Foothill College, Los Altos Hills, California

Randall W. Scott, Northern Arizona University, Flagstaff

David Seigler, University of Illinois, UrbanaChampaign

John C. Semple, University of Waterloo, Ontario, Canada

James R. Shevock, California Academy of Sciences, San Francisco

Teresa Sholars, College of the Redwoods, Fort Bragg, California

Leila M. Shultz, Utah State University, Logan

Michael Silveira, San Diego State University, California

Scott Simono, University of California, Berkeley

Beryl B. Simpson, The University of Texas at Austin

Michael G. Simpson, San Diego State University, California

Elizabeth M. Skendzic, Pacific Lutheran University, Tacoma, Washington

Mark W. Skinner, United States Department of Agriculture, Forest Service, Pacific Northwest Region, Portland, Oregon

Tracey Slotta, Montgomery College, Rockville, Maryland

Alan R. Smith, University of California, Berkeley

James P. Smith, Jr., Humboldt State University, Arcata, California

S. Galen Smith, University of WisconsinWhitewater; University of Wisconsin-Madison

Douglas E. Soltis, University of Florida, Gainesville

Pamela S. Soltis, University of Florida, Gainesville

Robert J. Soreng, Smithsonian Institution, Washington, District of Columbia

Valerie Soza, University of Washington, Seattle

Richard Spellenberg, New Mexico State University, Las Cruces

G. Ledyard Stebbins $\dagger$, University of California, Davis

Kelly Steele, Arizona State University, Mesa

Saša Stefanović, University of Toronto, Mississauga, Ontario, Canada

William J. Stone, University of California, Berkeley

Suzanne C. Strakosh, Kansas State University, Manhattan

John L. Strother, University of California, Berkeley 
Fosiée Tahbaz, University of California, Berkeley

Jennifer Talbot, Washington University, St. Louis, Missouri

David C. Tank, University of Idaho, Moscow

Sarah Taylor, The University of Texas at Austin

W. Carl Taylor, National Science Foundation, Arlington, Virginia

John W. Thieret†, Northern Kentucky University, Highland Heights

David M. Thompson, Rancho Santa Ana Botanic Garden, Claremont, California

Robert F. Thorne, Rancho Santa Ana Botanic Garden, Claremont, California

Steven L. Timbrook, Ganna Walska Lotusland Foundation, Santa Barbara

Ruth E. Timme, The University of Texas at Austin

Debra K. Trock, California Academy of Sciences, San Francisco

Arthur O. Tucker, Delaware State University, Dover

Gordon C. Tucker, Eastern Illinois University, Charleston

John M. Tucker†, University of California, Davis

Charles E. Turner†, United States Department of Agriculture, Agricultural Research Service, Albany, California

Lowell E. Urbatsch, Louisiana State University, Baton Rouge

Jesús Valdés-Reyna, Universidad Autónoma Agraria Antonio Narro, Saltillo, Coahuila, México

Henk van der Werff, Missouri Botanical Garden, St. Louis

Brian Vanden Heuvel, Colorado State University, Pueblo

Staria S. Vanderpool, Arkansas State University, Jonesboro

Michael C. Vasey, San Francisco State University, California

Michael A. Vincent, Miami University, Oxford, Ohio

Nancy J. Vivrette, Santa Barbara Botanic Garden, California

Linda Ann Vorobik, University of California, Berkeley

Piet Vorster, University of Stellenbosch, South Africa

Eric B. Wada, Folsom Lake College, California

Warren L. Wagner, Smithsonian Institution, Washington, District of Columbia

M. Andrew Walker, University of California, Davis

Gary D. Wallace, Rancho Santa Ana Botanic Garden, Claremont, California
Livia Wanntorp, Stockholm University, Sweden

Michael J. Warnock, University of Missouri, Columbia

Linda E. Watson, Miami University, Oxford, Ohio

Grady L. Webster†, University of California, Davis

Robert Webster, United States Department of Agriculture, United States National Arboretum, Washington, District of Columbia

Thomas L. Wendt, Colegio de Postgraduados, Chapingo, Mexico

Margriet Wetherwax, University of California, Berkeley

R. David Whetstone, Jacksonville State University, Alabama

Justen Whittall, Santa Clara University, California

Alan T. Whittemore, United States Department of Agriculture, United States National Arboretum, Washington, District of Columbia

Jeannette Whitton, University of British Columbia, Vancouver, Canada

Delbert Wiens, University of Utah, Salt Lake City

John H. Wiersema, United States Department of Agriculture, Agricultural Research Service, Beltsville, Maryland

Dieter H. Wilken, Santa Barbara Botanic Garden, California

Michael P. Williams, Butte College, Oroville, California

Carol A. Wilson, Rancho Santa Ana Botanic Garden and Claremont Graduate University, California

Paul Wilson, California State University, Northridge

Michael D. Windham, Duke University, Durham, North Carolina

Dennis W. Woodland, Andrews University, Berrien Springs, Michigan

Martin F. Wojciechowski, Arizona State University, Tempe

Steven J. Wolf, California State University Stanislaus, Turlock

Lindsay P. Woodruff, The University of Texas at Austin

Thomas Worley, Eureka, California

George Yatskievych, Missouri Botanical Garden, St. Louis

Elizabeth H. Zacharias, Harvard University, Cambridge, Massachusetts

Peter F. Zika, University of Washington, Seattle

Wendy B. Zomlefer, University of Georgia, Athens 